\title{
Esthetic perception of facial profile changes after extraction and nonextraction Class II treatment
}

\author{
Andréa Damasceno ROCHA ${ }^{(a)}$ \\ Cleomária Evelyn Vieira Freire \\ CASTELUCI(b) \\ Fernando Pedrin Carvalho \\ FERREIRA $^{(c)}$ iD \\ Ana Claudia CONTI(b) \\ Marcio Rodrigues ALMEIDA ${ }^{(b)}$ \\ Renata Rodrigues \\ ALMEIDA-PEDRIN ${ }^{(b)}$ \\ (a) Universidade do Sagrado Coração - \\ Unisagrado, Orthodontic Department, \\ Bauru, SP, Brazil. \\ (b) Universidade do Norte do Paraná - Unopar, \\ Orthodontic Department, Londrina, PR, Brazil. \\ (c) Private Orthodontics Office, Bauru, SP, Brazil.
}

Declaration of Interests: The authors certify that they have no commercial or associative interest that represents a conflict of interest in connection with the manuscript.

Corresponding Author:

Renata Rodrigues Almeida-Pedrin

E-mail: renatinhaalmeida@uol.com.br

https://doi.org/10.1590/1807-3107bor-2020.vol34.0003

Submitted: April 29, 2019

Accepted for publication: December 10, 2019

Last revision: January 10, 2020
Abstract: This retrospective study evaluated facial profile pleasantness determined by two protocols of Class II treatment. The sample comprised facial profile silhouettes obtained retrospectively from the pretreatment (T1) and posttreatment (T2) cephalograms of 60 patients (42 males and 18 females) divided into two groups. One group of 30 patients (mean age of 12.84 years) was treated with the extraction of maxillary first premolars (mean treatment time of 2.7 years), and the other group of 30 patients (mean age of 12.81 years) was treated with a mandibular advancement appliance (Forsus) (mean treatment time of 2.49 years). The facial profile silhouettes (T1 and T2) were randomly distributed in an album containing one patient per sheet. The examiners consisted of 60 orthodontists and 60 lay individuals, who analyzed the profiles in regard to facial pleasantness, using the Likert scale. A comparison between stages T1 and T2 of the two treatment protocols and between the examiners was performed by mixed-design analysis of variance at a significance level of $5 \%$. The results demonstrated a significant difference between T1 and T2 (greater scores for T2 compared to T1), and between lay individuals and orthodontists (orthodontists assigned higher scores), but with no significant difference between the treatment protocols. Both protocols produced positive effects on the facial profile esthetics, from the standpoint of lay individuals and orthodontists.

Keywords: Malocclusion, ; Angle Class II; Orthodontics, Corrective; Orthodontic Appliances, Functional; Tooth Extraction.

\section{Introduction}

Class II malocclusion is the most frequent sagittal discrepancy in orthodontic offices, presenting high prevalence (38\%) early in childhood, without presenting self-correction. ${ }^{1}$ Maxillary protrusion and/or mandibular retrusion are the etiologic factors involved in this discrepancy, leading to maxillomandibular sagittal step, increased overjet and convex facial profile. Several treatment protocols have been proposed depending on the location of the dysplasia, including headgear, appliances for maxillary molar distalization, mandibular anterior repositioning, extraction of premolars and mechanics with Class II elastics. ${ }^{2,3,4,5}$

For more than one century, Class II division 1 malocclusion has been treated with different types of functional appliances for mandibular 
advancement, considering the predominance of retrognathism in this malocclusion. For this reason, several mandibular anterior repositioning appliances are available in the market (Bionator, Herbst, Forsus, Twin-Force, etc.). ${ }^{6,78}$ The Forsus FRD appliance is a semi-rigid device that provides greater flexibility in mandibular positioning, thus making it better accepted by patients. The dentoalveolar effects produced by the therapy using Forsus promote sagittal correction of Class II malocclusion. The sagittal occlusal relationships are significantly enhanced with distal movement of maxillary molars and mesial movement of mandibular molars. The improvement in the facial profile is evidenced by changes in soft tissue cephalometric measurements with retrusion of the upper lip and protrusion of the lower lip.9

Considering the multifactorial etiology of this malocclusion, and the different treatment protocols suggested, it is interesting that there is no consensus among specialists regarding the best method to correct the discrepancy. Some authors believe that the systematic adoption of therapy with extraction may provide unfavorable results for the patient's facial esthetics. Analytically, some investigators have conducted studies to evaluate the effects of Class II treatment by premolar extraction on facial profile esthetics, and have found that this method does not seem to cause harmful effects on the facial profile. The effects were very similar on soft tissues, and the improvement in facial profile was more beneficial with the extraction of premolars than treatment without extraction. ${ }^{10,11,12}$

Today's growing interest in facial esthetics has been increasing the search for orthodontic treatment. As a result, modern orthodontics seeks advances not only for dental correction, but also for ways to improve facial esthetics. Facial attractiveness is positively correlated with self-esteem, and both interpersonal and professional relationships. ${ }^{13}$ Since beauty standards differ according to the perception of lay individuals and orthodontists, it is fundamental to know the opinion of both groups. Satisfaction with facial and dental appearance is a predictor for knowing patients' expectations toward orthodontic treatment. ${ }^{14}$

Several studies in the literature demonstrate the efficacy of the Forsus appliance and the protocol for correction of Class II malocclusion by extraction. 8,9,10,11 However, orthodontic planning should achieve dental corrections by considering the soft tissue responses not only in terms of the cephalometric numerical values, but especially the impact on the facial profile. Therefore, this study analyzed the facial pleasantness of patients treated with Forsus and with the extraction of maxillary premolars, according to orthodontists and lay individuals.

\section{Methodology}

This retrospective study was approved by the Research Ethics Committee of the Sagrado Coração University, under protocol no. 2.138.340.

The sample calculation of the number of patients required for each group was based on a study by Pithon et al., ${ }^{15}$ in which the greatest standard deviation of scores assigned was 1.25. Thus, considering a significance level of $5 \%$ and power of $80 \%$, with a minimum difference of 1.0 between the two groups, a minimum sample of 26 patients was calculated for each group. As for the number of evaluators, a comparison performed by a paired model, considering a significance level of $5 \%$ and a power of $80 \%$ to detect a minimum difference of 0.5 between the two groups, revealed a minimum of 51 examiners in each group.

The study sample comprised 60 patients treated by corrective orthodontics; one group of 30 patients was treated by extraction of maxillary first premolars, and the other group of 30 patients was treated with the Forsus appliance (3M Unitek, Monrovia, CA, USA). The following inclusion criteria were observed for sample selection: patients presenting initial and final lateral cephalograms; presence of bilateral Class II division 1 malocclusion; absence of hypodontia or permanent tooth loss, except for the third molars; absence of supernumerary or impacted teeth, or dental anomalies of size and/or shape; absence of mandibular crowding; convex facial profile; and increased overjet.

The groups were compatible concerning gender, initial and final age, treatment time and cephalometric measurements (Table 1). In the extraction group, patients were treated by extraction of maxillary first premolars. This group was composed of 20 males 
(66.7\%) and 10 females (33.3\%), with an initial mean age of 12.84 years, and a final mean age of 15.52 years (Table 1). The patients were treated in a private office, with preadjusted fixed orthodontic appliance slot.022" x.028", using only a transpalatal arch (TPA) as anchorage (Figure 1). The retraction of anterior teeth was performed using sliding mechanics, with chain elastics from the mesial surface of the maxillary canines to the tube on the maxillary first molars. Class II elastics were used to enhance the correction of malocclusion. The mean treatment time was 2.7 years (32.4 months).

The patients of the Forsus group were treated at the Post Graduation Clinic of the Universidade

Table 1. Comparison of characteristics of the sample in the two treatment protocols performed by independent t-test.

\begin{tabular}{|c|c|c|c|}
\hline Characteristic & Extraction & Forsus & $p$-value \\
\hline \multicolumn{4}{|l|}{ Gender } \\
\hline \multicolumn{4}{|l|}{ Male } \\
\hline $\mathrm{n}$ & 20 & 22 & \multirow{5}{*}{$0.573 \mathrm{~ns}$} \\
\hline$\%$ & 66.7 & 73.3 & \\
\hline Female & & & \\
\hline $\mathrm{n}$ & 10 & 8 & \\
\hline$\%$ & 33.3 & 26.7 & \\
\hline \multicolumn{4}{|l|}{ Initial age } \\
\hline Mean & 12.84 & 12.82 & \multirow{2}{*}{$0.970 \mathrm{~ns}$} \\
\hline SD & 2.09 & 1.95 & \\
\hline \multicolumn{4}{|l|}{ Final age } \\
\hline Mean & 15.52 & 15.31 & \multirow{2}{*}{$0.716 \mathrm{~ns}$} \\
\hline SD & 2.35 & 2.09 & \\
\hline \multicolumn{4}{|l|}{ Treatment time } \\
\hline Mean & 2.70 & 2.49 & \multirow{2}{*}{$0.355 \mathrm{~ns}$} \\
\hline SD & 0.66 & 1.04 & \\
\hline \multicolumn{4}{|l|}{ Overiet } \\
\hline Mean & 6.05 & 5.87 & \multirow{2}{*}{$0.133 \mathrm{~ns}$} \\
\hline SD & 2.98 & 2.81 & \\
\hline \multicolumn{4}{|l|}{$\mathrm{SNA}^{\circ}$} \\
\hline Mean & 84.57 & 84.93 & \multirow{2}{*}{$0.727 \mathrm{~ns}$} \\
\hline SD & 3.87 & 4.07 & \\
\hline \multicolumn{4}{|l|}{$\mathrm{SNB}^{\circ}$} \\
\hline Mean & 78.58 & 78.91 & \multirow{2}{*}{$0.728 \mathrm{~ns}$} \\
\hline SD & 3.56 & 3.75 & \\
\hline \multicolumn{4}{|l|}{$\mathrm{ANB}^{\circ}$} \\
\hline Mean & 5.99 & 6.02 & \multirow{2}{*}{$0.967 \mathrm{~ns}$} \\
\hline SD & 2.77 & 2.86 & \\
\hline
\end{tabular}

ns: statistically non-significant difference.
Sagrado Coração, and comprised 22 males (73.3\%) and 8 females $(26.7 \%)$, with an initial mean age of 12.82 years and a final mean age of 15.31 years (Figure 1). They were treated with preadjusted fixed orthodontic appliance slot.022" x.028". The Forsus appliance was installed after leveling and aligning.019" x.025" stainless steel archwires placed on the maxillary and mandibular arches. Class II elastics were used to enhance the malocclusion correction. The mean treatment time with the Forsus appliance was 6.94 months, and the total treatment time associated with corrective orthodontics was 2.49 years (29.9 months).

The soft tissue profile tracings were obtained from lateral cephalograms pre- (T1) and posttreatment (T2) for each patient, by a single investigator (A.D.R) in a dark room, to facilitate the identification of anatomical structures. The soft tissue outline was traced with a $0.5 \mathrm{~mm}$ HB pencil on an Ultraphan paper sheet 0.07-mm thick and $17.5-\mathrm{mm}$ wide and $17.5-\mathrm{mm}$ long, adapted to the 120 cephalograms. The tracings were scanned and transferred to a computer, and the facial profile silhouettes were filled on Adobe Photoshop CC2018* software (Adobe Systems, San Jose, USA) by a single computer technician. The two profile silhouettes (pre- and posttreatment) of each patient were randomly inserted on the same sheet, i.e., the pretreatment profile could be on the right or left of the page, producing an album with the 120 silhouettes of the 60 patients. Two groups of evaluators participated in the study to score the profiles of each patient. The group of lay individuals was composed of 60 individuals without any knowledge of dentistry, 30 males (50\%) and 30 females (50\%), with a mean age of 32.46 years. The group of orthodontists included 60

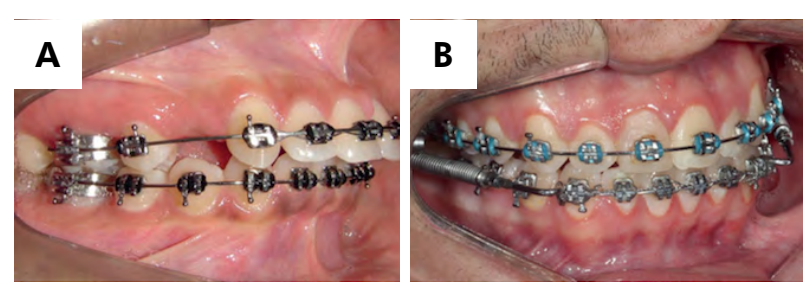

Figure 1. A. Intraoral lateral view of a patient from the extraction group. B. Intraoral lateral view of a patient from the Forsus group. 
specialists, 28 females (46.7\%) and 32 males (53.3\%), with a mean age of 40.72 years.

Each evaluator received an album containing the profile silhouettes of the 60 patients, and instructions on how to fill out the scores on pleasantness. The examiners were requested to score the facial profiles (A and B) using a Likert scale, as follows: 1- very unpleasant, 2- unpleasant, 3- acceptable, 4- pleasant and 5 - very pleasant. The examiner had to write an " $x$ " on the boxes corresponding to both profiles (Figure 2).

\section{Statistical analysis}

The scores assigned to facial profile pleasantness were described as means and standard deviations. All measurements passed the Kolmogorov-Smirnov normality test.

Comparison of the characteristics of the sample for the two treatment protocols was performed with an independent t-test. Comparison between the protocols (extraction versus Forsus), stages (T1 and T2) and evaluators (lay individuals and orthodontists) was performed by three-way mixed-model analysis of variance. All tests were applied at a significance level of $5 \%$. All statistical procedures were performed using SPSS Statistics software version 25 (SPSS, Chicago, USA).

\section{Agreement test}

The 120 profile silhouettes were re-examined by $30 \%$ of the lay individuals and $30 \%$ of the orthodontists, both selected randomly, three weeks after the first evaluation. The intraexaminer agreement was calculated by the weighed kappa. The kappa statistics revealed values of 0.56 for lay individuals and 0.61 for orthodontists. According to Landis and Koch, ${ }^{16}$ the agreement was considered moderate for lay individuals and substantial for orthodontists.

\section{Results}

The results revealed no significant difference between the extraction and the Forsus treatment protocols. There was significant difference between stages T1 and T2 (with greater scores at T2 than T1), for both protocols analyzed. Concerning the group of examiners, there was significant difference between lay individuals and orthodontists (with greater scores given by orthodontists than lay individuals).

\section{Discussion}

Orthodontic treatment has been using a more therapeutic approach in its ongoing search to enhance

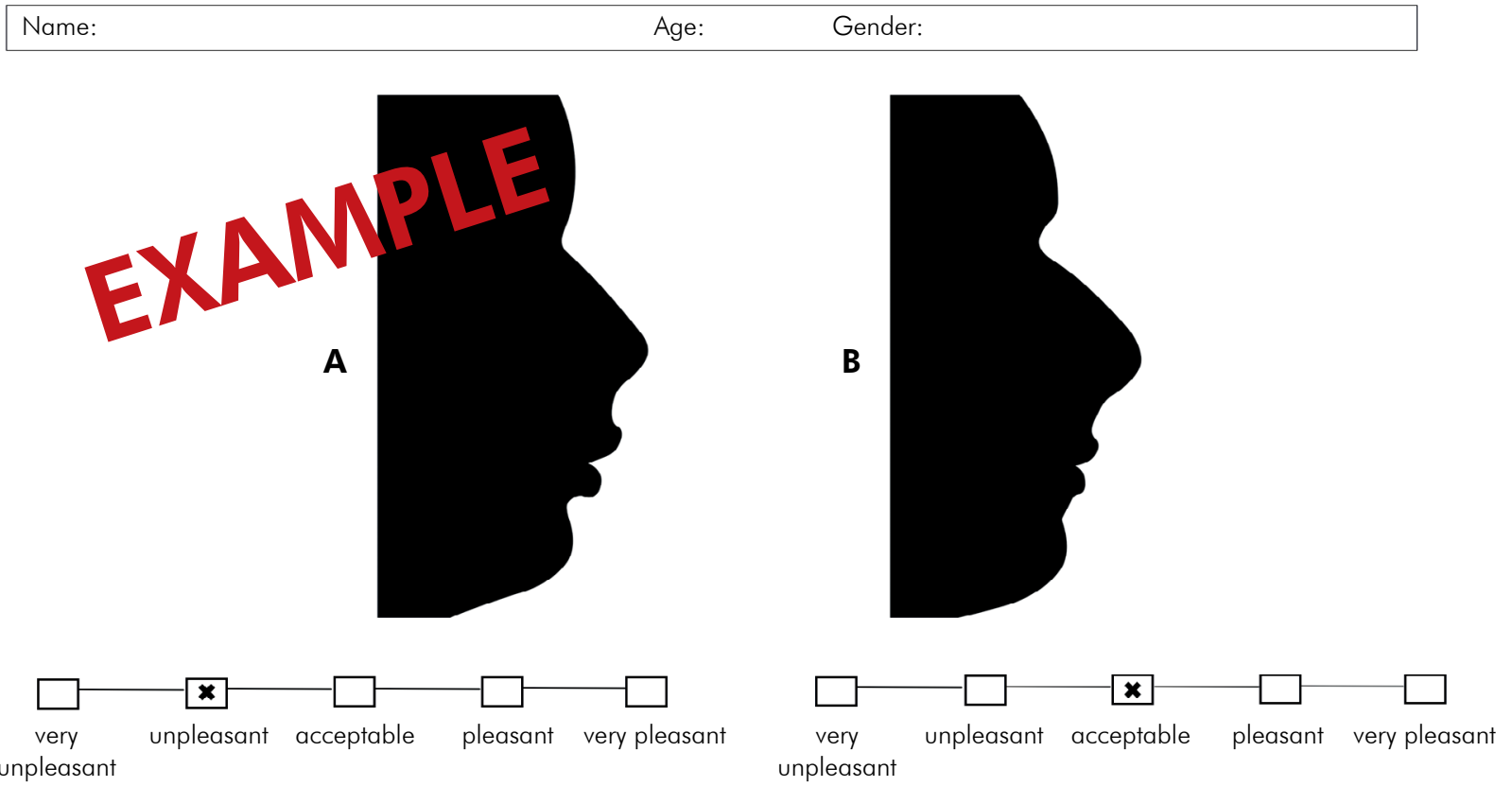

Figure 2. Example sheet for evaluation of the profile pleasantness using the Likert scale. 
facial esthetics, particularly one not solely restricted to dental corrections. The present investigation used an original method to perform a subjective analysis based on comparing the facial profile esthetics of patients with Class II malocclusion treated with the Forsus appliance versus extraction of maxillary premolars.

The literature presents several studies related to determining facial attractiveness, based on cephalometric measurements. ${ }^{4,6,9,12}$ However, it is interesting to analyze whether a certain treatment protocol may offer more benefits for enhancing attractiveness, in the opinion of both lay individuals and orthodontists, considering that beauty standards range according to the individual viewpoint of the observer. Some investigators have used photographs to analyze facial esthetics, basing the outcome of a pleasant face on several aspects, including color of skin and eyes, hair, age and ethnicity. ${ }^{14,17,18}$ However, aspects that influence examiners' perceptions of facial attractiveness may be eliminated by using profile silhouettes. ${ }^{15,19,20,21,22}$

The concept that orthodontic treatment by extraction of premolars damages facial profile esthetics often keeps the orthodontist back from choosing this option in devising the treatment planning. In this respect, over the years, orthodontists have been investigating the relationship between facial profile esthetics and orthodontic treatment by tooth extraction. 2,10,23,24,25 The results of the present study revealed no significant difference between the protocols using extraction versus a mandibular advancement appliance (Table 2), i.e., both treatment protocols provided positive changes to the patients' faces (Table 3).

The mean scores were higher for posttreatment in the group treated with extraction of premolars than pretreatment (Table 3). Therefore, the possible soft tissue changes promoted by the protocol with extraction proved extremely beneficial to the facial profile esthetics. Similar results were found in studies analyzing the soft tissue profile after orthodontic treatment with extraction. ${ }^{11,20,23,24}$

Conversely, it has been reported that the greatest benefit of treatment by extraction is achieved in cases with greater dental protrusion, marked crowding, and thick and protruded lips. ${ }^{10,11,26}$ However, in individuals presenting a thin upper lip and an increased nasolabial angle at pretreatment, extraction may affect the upper lip positioning negatively and increase the nasolabial angle. ${ }^{27}$

Table 2. Three-way mixed-model analysis of variance for comparison of scores assigned according to the treatment protocols.

\begin{tabular}{|c|c|c|c|c|c|c|c|c|}
\hline \multirow{2}{*}{ Stage } & \multirow{2}{*}{ Evaluator } & \multicolumn{2}{|c|}{ Extraction } & \multicolumn{2}{|c|}{ Forsus } & \multicolumn{2}{|c|}{ diff. } & \multirow{2}{*}{$\mathrm{p}$-value } \\
\hline & & Mean & SD & Mean & SD & Mean & $\%$ & \\
\hline \multirow{2}{*}{$\mathrm{Tl}$} & Lay & 2.36 & 0.48 & 2.37 & 0.49 & 0.02 & 0.7 & \multirow{4}{*}{$0.644 \mathrm{~ns}$} \\
\hline & Ortho & 2.43 & 0.68 & 2.47 & 0.71 & 0.04 & 1.6 & \\
\hline \multirow{2}{*}{$\mathrm{T} 2$} & Lay & 2.52 & 0.49 & 2.61 & 0.42 & 0.08 & 3.3 & \\
\hline & Ortho & 2.64 & 0.61 & 2.73 & 0.64 & 0.10 & 3.7 & \\
\hline Total & & 2.49 & 0.57 & 2.55 & 0.58 & 0.06 & 2.4 & \\
\hline
\end{tabular}

ns: statistically non-significant difference.

Table 3. Comparison of scores assigned according to the treatment stages performed by three-way mixed-model analysis of variance.

\begin{tabular}{|c|c|c|c|c|c|c|c|c|}
\hline \multirow{2}{*}{ Protocol } & \multirow{2}{*}{ Evaluator } & \multicolumn{2}{|c|}{$\mathrm{Tl}$} & \multicolumn{2}{|c|}{$\mathrm{T} 2$} & \multicolumn{2}{|c|}{ diff. } & \multirow{2}{*}{$\mathrm{p}$-value } \\
\hline & & Mean & SD & Mean & SD & Mean & $\%$ & \\
\hline \multirow{2}{*}{ Extraction } & Lay & 2.36 & 0.48 & 2.52 & 0.49 & 0.16 & 7.0 & \multirow{4}{*}{0.002} \\
\hline & Ortho & 2.43 & 0.68 & 2.64 & 0.61 & 0.21 & 8.7 & \\
\hline \multirow{2}{*}{ Forsus } & Lay & 2.37 & 0.49 & 2.61 & 0.42 & 0.23 & 9.8 & \\
\hline & Ortho & 2.47 & 0.71 & 2.73 & 0.64 & 0.27 & 10.9 & \\
\hline Total & & 2.41 & 0.60 & 2.63 & 0.55 & 0.22 & 9.1 & \\
\hline
\end{tabular}

*statistically significant difference $(p<0.05)$. 
Studies analyzing the long-term influence of treatment by extraction on facial esthetics concluded that this treatment does not negatively influence facial attractiveness in Class II malocclusions. ${ }^{23,24,26,28}$ Just like the extraction group, treatment with the Forsus appliance also produced positive changes in the facial profile; however, both groups of evaluators assigned greater scores to the Forsus appliance at posttreatment (Table 3), thus corroborating other studies that used fixed functional appliances to achieve an improvement in the facial profile. ${ }^{4,21,29}$ Even though the protocols for both extraction and Forsus achieved favorable results, the observation between lay individuals and orthodontists was different concerning the facial impact, in that the impact was more perceptible to orthodontists. Other studies ${ }^{21,22,29}$ also observed differences in the opinion of lay individuals and orthodontists.

The results demonstrated a statistically significant difference $(p=0.003)$ in the opinion of lay individuals and orthodontists, even though both evaluated the posttreatment profile as more pleasant (Table 4). This corroborates the study by Almeida-Pedrin et al. ${ }^{20}$, who analyzed the facial profile changes promoted by orthodontic treatment with the extraction of two maxillary first premolars, and revealed that 83 out of 90 examiners preferred the posttreatment profile.

As corroborated by their study, the investigations made into facial attractiveness by evaluating the soft tissue profile have demonstrated that the differences in the outcomes are viewed differently by orthodontists and lay individuals. ${ }^{9,21,22}$ An evaluation by Paula et al. ${ }^{21}$ of the treatment effects achieved by the mandibular protraction appliance (MPA) on the profile silhouettes showed that there was a difference in the perception of these effects by examiners. They found that lay individuals identified the difference between the pre- and posttreatment silhouettes as being greater than the orthodontists, unlike the present study, in which the orthodontists assigned greater scores to the posttreatment outlines. This discordance might be attributed to the different methodology applied. Despite this disagreement, the groups of examiners in both studies identified improvements in the posttreatment profile.

Likewise, Rego et al..$^{29}$ reported that lay individuals quantified soft tissue changes as greater after treatment with the Herbst appliance. However, in the present study, the results presented in Table 4 reveal a significant difference between the examiners $(p=0.003)$, in that the orthodontists were more permissive in their evaluation, by assigning greater scores to the pleasantness of facial profile. This may be explained by the technical knowledge of professionals, since orthodontists understand how difficult it is to achieve skeletal changes in orthodontic treatments.

Among all the aspects analyzed in this study, the most important result was surely that of certifying that both protocols produced a positive effect on facial profile esthetics, and that the mean posttreatment scores were higher for both groups of examiners. It should be highlighted that the improvement in facial profile may also be attributed to the natural growth of the individuals analyzed.

Therefore, both treatments ultimately led to a noticeable improvement in facial pleasantness, considering that the patients were scored as having unpleasant/acceptable faces in stage T1 (pretreatment), and that this score improved substantially in stage

Table 4. Three-way mixed-model analysis of variance for comparison of scores assigned according to the category of evaluators.

\begin{tabular}{|c|c|c|c|c|c|c|c|c|}
\hline \multirow{2}{*}{ Protocol } & \multirow{2}{*}{ Stage } & \multicolumn{2}{|c|}{ Lay } & \multicolumn{2}{|c|}{ Ortho } & \multicolumn{2}{|c|}{ diff. } & \multirow{2}{*}{$p$-value } \\
\hline & & Mean & SD & Mean & $\mathrm{SD}$ & Mean & $\%$ & \\
\hline \multirow{2}{*}{ Extraction } & $\mathrm{T} 1$ & 2.36 & 0.48 & 2.43 & 0.68 & 0.07 & 2.9 & \multirow{4}{*}{$0.003^{*}$} \\
\hline & $\mathrm{T} 2$ & 2.52 & 0.49 & 2.64 & 0.61 & 0.12 & 4.6 & \\
\hline \multirow{2}{*}{ Forsus } & $\mathrm{T} 1$ & 2.37 & 0.49 & 2.47 & 0.71 & 0.09 & 3.8 & \\
\hline & T2 & 2.61 & 0.42 & 2.73 & 0.64 & 0.13 & 4.9 & \\
\hline Total & & 2.47 & 0.47 & 2.57 & 0.66 & 0.10 & 4.1 & \\
\hline
\end{tabular}

*statistically significant difference $(p<0.05)$. 
T2 (posttreatment), when the profile silhouettes were closer to acceptable.

\section{Conclusions}

The orthodontic treatment of Class II division 1 malocclusion both with extraction of maxillary first premolars and with the Forsus appliance had positive effects on facial profile esthetics, as evaluated by both lay examiners and orthodontists, who assigned greater scores to the posttreatment profile.

Both treatment protocols produced similar results on the facial profile esthetics.

The group of orthodontists assigned higher scores to both pre- and posttreatment profiles than lay individuals.

\section{References}

1. Almeida MR, Pereira AL, Almeida RR, Almeida-Pedrin RR, Silva Filho OG. Prevalence of malocclusion in children aged 7 to 12 years. Dental Press J Orthod. 2011;16(4):123-31. https://doi.org/10.1590/S2176-94512011000400019

2. Khan M, Fida M. Soft tissue profile response in extraction versus non-extraction orthodontic treatment. J Coll Physicians Surg Pak. 2010 Jul;20(7):454-9.

3. Bondemark L, Karlsson I. Extraoral vs intraoral appliance for distal movement of maxillary first molars: a randomized controlled trial. Angle Orthod. 2005 Sep;75(5):699-706. https://doi.org/10.1043/0003-3219(2005)75[699:EVIAFD]2.0.CO;2

4. Bilgiç F, Başaran G, Hamamci O. Comparison of Forsus FRD EZ and Andresen activator in the treatment of class II, division 1 malocclusions. Clin Oral Investig. 2015 Mar;19(2):445-51. https://doi.org/10.1007/s00784-014-1237-y

5. Jones G, Buschang PH, Kim KB, Oliver DR. Class II non-extraction patients treated with the Forsus Fatigue Resistant Device versus intermaxillary elastics. Angle Orthod. 2008 Mar;78(2):332-8. https://doi.org/10.2319/030607-115.1

6. Perinetti G, Primožič J, Furlani G, Franchi L, Contardo L. Treatment effects of fixed functional appliances alone or in combination with multibracket appliances: A systematic review and meta-analysis. Angle Orthod. 2015 May;85(3):480-92. https://doi.org/10.2319/102813-790.1

7. Moro A, Borges SW, Spada PP, Morais ND, Correr GM, Chaves CM Jr, et al. Twenty-year clinical experience with fixed functional appliances. Dental Press J Orthod. 2018 Mar-Apr;23(2):87-109. https://doi.org/10.1590/2177-6709.23.2.087-109.sar

8. Pacha MM, Fleming PS, Johal A. A comparison of the efficacy of fixed versus removable functional appliances in children with Class II malocclusion: A systematic review. Eur J Orthod. 2016 Dec;38(6):621-30. https://doi.org/10.1093/ejo/cjv086

9. Franchi L, Alvetro L, Giuntini V, Masucci C, Defraia E, Baccetti T. Effectiveness of comprehensive fixed appliance treatment used with the Forsus Fatigue Resistant Device in Class II patients. Angle Orthod. 2011 Jul;81 (4):678-83. https://doi.org/10.2319/102710-629.1

10. Bishara SE, Cummins DM, Jakobsen JR, Zaher AR. Dentofacial and soft tissue changes in Class II, division 1 cases treated with and without extractions. Am J Orthod Dentofacial Orthop. 1995 Jan;107(1):28-37. https://doi.org/10.1016/S0889-5406(95)70154-0

11. Lim HJ, Ko KT, Hwang HS. Esthetic impact of premolar extraction and nonextraction treatments on Korean borderline patients. Am J Orthod Dentofacial Orthop. 2008 Apr;133(4):524-31. https://doi.org/10.1016/i.ajodo.2006.04.051

12. Janson G, Castello Branco N, Aliaga-Del Castillo A, Henriques JF, Morais JF. Soft tissue treatment changes with fixed functional appliances and with maxillary premolar extraction in Class II division 1 malocclusion patients. Eur J Orthod. 2018 Apr;40(2):214-22. https://doi.org/10.1093/ejo/cjx053

13. Wezel NA, Bos A, Prahl C. Expectations of treatment and satisfaction with dentofacial appearance in patients applying for orthodontic treatment. Am J Orthod Dentofacial Orthop. 2015 Jun;147(6):698-703. https://doi.org/10.1016/i.ajodo.2015.01.024

14. Kiekens RM, Maltha JC, Hof MA, Straatman H, Kuijpers-Jagtman AM. Panel perception of change in facial aesthetics following orthodontic treatment in adolescents. Eur J Orthod. 2008 Apr;30(2):141-6. https://doi.org/10.1093/ejo/cjml14

15. Pithon MM, Lacerda-Santos R, Oliveira DL, Alves JV, Britto JP, Souza ES, et al. Esthetic perception of facial profile after treatment with the Thurow Appliance. Braz Oral Res. 2015;29(1):1-7. https://doi.org/10.1590/1807-3107BOR-2015.vol29.0043

16. Landis JR, Koch GG. The measurement of observer agreement for categorical data. Biometrics. 1977 Mar;33(1):159-74. https://doi.org/10.2307/2529310

17. Almeida MD, Farias AC, Bittencourt MA. Influence of mandibular sagittal position on facial esthetics. Dental Press J Orthod. 2010;15(2):87-96. https://doi.org/10.1590/S2176-94512010000200012.

18. Spyropoulos MN, Halazonetis DJ. Significance of the soft tissue profile on facial esthetics. Am J Orthod Dentofacial Orthop. 2001 May;119(5):464-71. https://doi.org/10.1067/mod.2001.113656

19. Hockley A, Weinstein M, Borislow AJ, Braitman LE. Photos vs silhovettes for evaluation of African American profile esthetics. Am J Orthod Dentofacial Orthop. 2012 Feb;141(2):161-8. https://doi.org/10.1016/j.ajodo.2011.06.041 
Esthetic perception of facial profile changes after extraction and nonextraction Class II treatment

20. Almeida-Pedrin RR, Guimarães LB, Almeida MR, Almeida RR, Ferreira FP. Assessment of facial profile changes in patients treated with maxillary premolar extractions. Dental Press J Orthod. 2012;17(5):131-7. https://doi.org/10.1590/S2176-94512012000500018

21. Paula ECM, Conti ACCF, Siqueira DF, Valarelli DP, Almeida-Pedrin RR. Esthetic perceptions of facial silhouettes after treatment with a mandibular protraction appliance. Am J Orthod Dentofacial Orthop. 2017 Feb;151(2):311-6. https://doi.org/10.1016/i.ajodo.2016.06.038

22. Barroso MC, Silva NC, Quintão CC, Normando D. The ability of orthodontists and laypeople to discriminate mandibular stepwise advancements in a Class II retrognathic mandible. Prog Orthod. 2012 Sep;13(2):141-7. https://doi.org/10.1016/i.pio.2011.12.001

23. Seben MP, Valarelli FP, Freitas KM, Cançado RH, Bittencourt Neto AC. Cephalometric changes in Class II division 1 patients treated with two maxillary premolars extraction. Dental Press J Orthod. 2013 Jul-Aug;18(4):61-9. https://doi.org/10.1590/S2176-94512013000400010

24. Janson G, Junqueira CH, Mendes LM, Garib DG. Influence of premolar extractions on long-term adult facial aesthetics and apparent age. Eur J Orthod. 2016 Jun;38(3):272-80. https://doi.org/10.1093/ejo/cjv039

25. Basciftci FA, Usumez S. Effects of extraction and nonextraction treatment on class I and class II subjects. Angle Orthod. 2003 Feb;73(1):36-42.

26. lared W, Silva EMK, lared W, Macedo CR. Esthetic perception of changes in facial profile resulting from orthodontic treatment with extraction of premolars: A systematic review. J Am Dent Assoc. 2017 Jan;148(1):9-16. https://doi.org/10.1016/j.adaj.2016.09.004

27. Tadic N, Woods MG. Incisal and soft tissue effects of maxillary premolar extraction in class II treatment. Angle Orthod. 2007 Sep;77(5):80816. https://doi.org/10.2319/081706-336

28. McGuinness NJ, Burden DJ, Hunt OT, Johnston CD, Stevenson M. Long-term occlusal and soft-tissue profile outcomes after treatment of Class II Division 1 malocclusion with fixed appliances. Am J Orthod Dentofacial Orthop. 2011 Mar;139(3):362-8. https://doi.org/10.1016/i.ajodo.2009.05.035

29. Rego MV, Martinez EF, Coelho RM, Leal LM, Thiesen G. Perception of changes in soft-tissue profile after Herbst appliance treatment of Class II Division 1 malocclusion. Am J Orthod Dentofacial Orthop. 2017 Mar;151(3):559-64. https://doi.org/10.1016/i.ajodo.2016.08.028 